$(\mathrm{p}<0.001)$. Partnership mixing on age group, race/ethnicity, and military status each showed statistically significant difference by partnership type (MSM, MSW, WSM, WSW) $(\mathrm{p}<0.001)$. Recent STI was not associated with disassortative mixing on age group or military status but was associated with disassortative mixing on race/ethnicity $(p=0.003) .194$ of 669 (29.0\%) subjects reported concurrent partnerships or suspected sexual concurrency by their partner(s). Suspected sexual concurrency by respondents' partner(s) significantly increased the odds of STI in the respondent after adjusting for partnership type $(p=0.02)$.

Military service includes unique social and cultural constructs that may influence sexual partnership formation. While not likely representative of the U.S. military as a whole, these results attempt to identify partnership characteristics and risk factors that may support the maintenance of high rates of select STIs among military populations.

\section{P291 GENOMIC EPIDEMIOLOGY OF NEISSERIA GONORRHOEAE ISOLATES IN SWEDEN - 2016 NATIONAL STUDY}

\begin{abstract}
${ }^{1,2,3} \mathrm{R}$ Hadad*, ${ }^{1,2,3} \mathrm{D}$ Golparian, ${ }^{4}$ I Velicko, ${ }^{5} \mathrm{Y}$ Lindroth, ${ }^{4} \mathrm{~A}$ Ohlsson, ${ }^{1,2,3} \mathrm{H}$ Fredlund, 1,2,3 M Unemo. 'WHO Collaborating Centre for Gonorrhoea and other Sexually Transmitted Infections, Örebro, Sweden; ${ }^{2}$ National Reference Laboratory for STIS, Department of Laboratory Medicine, Örebro, Sweden; ${ }^{3}$ Faculty of Medicine and Health, Örebro University, Örebro, Sweden; ${ }^{4}$ Department of Clinical Microbiology, Karolinska University Hospital, Stockholm, Sweden; ${ }^{5}$ Department of Laboratory Medicine, Medical Microbiology, Lund University, Skåne Laboratory Medicine, Lund, Sweden
\end{abstract}

\subsection{6/sextrans-2021-sti.353}

Background The number of reported cases of gonorrhoea in Sweden continuously increased from an incidence of 7.8 per 100000 inhabitants in 2009 to 31.4 in 2019. The largest increase in incidence was observed during 2016-2017. No national molecular epidemiological study investigating the population of N. gonorrhoeae circulating in Sweden has been performed in the last two decades. Our aim was to examine the antimicrobial resistance (AMR) and genome-based epidemiology, in conjunction to patient epidemiological data, of all gonococcal isolates $(n=1279$; one isolate per case) from gonorrhoea cases in Sweden during 2016.

Methods AMR testing was performed using Etest, and MICs were interpreted using current clinical resistance breakpoints from EUCAST. All isolates were whole genome sequenced using Illumina HiSeq X platform. Patient epidemiological data was obtained from the Public Health Agency of Sweden.

Results The gonorrhoea patients consisted of 252 (19.7\%) women and 1027 men (80.3\%). The medium age of the women was 27.4 years and of the men 32.1 years. Regarding sexual orientation, $619(48.4 \%)$ reported homosexual, 605 (47.3\%) heterosexual, 31 (2.4\%) bisexual, and $24(1.9 \%) \mathrm{did}$ not report. Most prevalent countries of infection were Sweden $(n=875,68.4 \%)$, followed by Thailand $(n=70,5.5 \%)$ and Germany ( $\mathrm{n}=32,2.5 \%)$.

Overall, the phenotypic AMR was as follows: ceftriaxone and spectinomycin $(0 \%)$, cefixime $(1.7 \%)$, azithromycin $(1.3 \%)$ and ciprofloxacin (51.1\%). A high concordance between phenotypic AMR and molecular AMR determinants was found. Results from the genome-based epidemiology are currently in final analysis.
Conclusions AMR in N. gonorrhoeae in Sweden remains low, in particular to ceftriaxone and azithromycin that is recommended internationally for dual therapy. The incidence increases in Sweden appear to be driven by increased spread among men-who-have-sex-with-men but also younger heterosexuals of both genders. This is the first national genomebased epidemiological study for $\mathrm{N}$. gonorrhoeae in Sweden and final genomic results are pending.

\section{P292 ONLINE HIV/STI-CLINICAL TRAINING FOR ELEVEN PACIFIC ISLAND COUNTRIES PROVIDED BY THE UNDP MULTI-COUNTRY WESTERN PACIFIC INTEGRATED HIVI TB PROGRAMME}

${ }^{1} \mathrm{C}$ O'Connor*, ${ }^{2} \mathrm{~J}$ Cati, ${ }^{3} \mathrm{P}$ Maharaj, ${ }^{3} \mathrm{P}$ Sami, ${ }^{4} \mathrm{~T}$ Faletoese-Su'a, ${ }^{3} \mathrm{G}$ Tovmasyan. ${ }^{1}$ Kirby Institute, University of New South Wales, Sydney, Australia; ${ }^{2} F J N+$, Suva, Fiji; ${ }^{3}$ UNDPWestern Pacific, Suva, Fiji; ${ }^{4}$ UNDP-Western Pacific, Apia, Samoa

\subsection{6/sextrans-2021-sti.354}

Background The Pacific Islands have a low HIV prevalence, but high rates of STI's and large high-risk populations. The UNDP Programme supports 11 Pacific Island Countries (PICs). In-country clinical training are provided as well as HIV/TB diagnostics, treatments, and specialist HIV clinical advice. In 2020, in-country support was not possible because of the COVID19 pandemic.

Approach After discussion with UNDP partners and PICs HIV/ STI-healthcare workers, we presented the following online education:

- Monthly webinars. Eight one-hour webinars on Zoom, each repeated 4 hours later as the PICs span 7 time-zones. These case-based educational webinars covered HIV/STI clinical care related topics. Guest speakers were invited for specialist topics.

- In-country HIV/STI-online education workshops run over one-day for nine PICs and over half-day for Niue and Tuvalu, the two smallest PICs. The workshops ran in late 2020 to build on the webinar knowledge and were tailored to incountry needs. Training was mainly case based, co-facilitated by a worker from the Fijian HIV-positive peoples NGO, FJN + , with guest speakers invited for specialist topics.

\section{Outcomes}

- Monthly webinars: 323 individual attendances for the 8 webinars. 114 evaluations were returned; 95\% found the HIV webinars very helpful and 92\% would recommend them to other clinical staff. As poor internet was a problem, the presentation slides and the recorded webinars were sent to the PIC attendees.

- In-country online HIV/STI-education workshops: 241 participants across the PICs. A planning meeting was conducted with each PIC. The average scores in questionnaires pre- and post-training doubled from $40 \%$ to $80 \%$. Participants found the case-based training useful and requested follow-up training.

Innovation/Significance This is first time in the HIV/STI clinical support to the Pacific has been provided on-line and despite internet challenges, it is an economical and efficient way to provide ongoing HIV/STI clinical education in this remote setting. 